\title{
The combined use of embryos and semen for cryogenic conservation of mammalian livestock genetic resources
}

\author{
Paul J. BoetTcheR ${ }^{\mathrm{a}, \mathrm{b} *}$, Alessandra STELla ${ }^{\mathrm{c}}$, Flavia PIZZI ${ }^{\mathrm{a}}$, \\ Gustavo GANDINI $^{\mathrm{d}}$

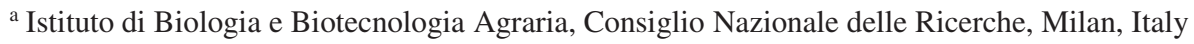 \\ ${ }^{\mathrm{b}}$ IAEA/FAO Joint Division for Nuclear Techniques in Food and Agriculture, Vienna, Austria \\ ${ }^{\mathrm{c}}$ CERSA-Fondazione Parco Tecnologico Padano Lodi, Italy \\ ${ }^{\mathrm{d}}$ Dipartimento di Scienze e Tecnologie Veterinarie per la Sicurezza Alimentare, Università \\ degli Studi di Milano, Milan, Italy
}

(Received 15 February 2005; accepted 6 July 2005)

\begin{abstract}
The objective of this empirical simulation study was to evaluate the use of a combination of semen and embryos in the creation of gene banks for reconstruction of an extinct breed. Such an approach was compared for banks with varying proportions of embryos on the basis of the amount of the material to be stored, time for reconstruction, maintenance of genetic variability, and probability of failure during reconstruction. Four types of populations were simulated, based on reproductive rate: single offspring, twinning, enhanced reproduction, and litter bearing. Reconstruction was simulated for banks consisting of different combinations of semen and reduced numbers of embryos (expressed as a percentage of the material needed for a bank containing exclusively embryos and ranging from 10 to 90\%). The use of a combination of semen and embryos increased the number of insemination cycles needed for reconstruction and the level of genetic relatedness in the reconstructed population. The risk for extinction was unacceptably high when a very low proportion of embryos $(<20 \%)$ was used. However, combining semen with embryos could decrease costs, allowing for the conservation of more breeds, and specific strategies for semen use could decrease the level of relationships in the reconstructed breed.
\end{abstract}

genetic conservation / breed reconstruction / gene banks / semen / embryo

\section{INTRODUCTION}

In 1992 the Convention on Biological Diversity advised ex situ conservation as an essential activity complementary to in situ conservation of farm animal genetic resources. Recently, disease outbreaks in Europe, whose control may

${ }^{*}$ Corresponding author: boettch@ibba.cnr.it 
require severe culling of the population, has underlined the importance of having cryopreserved genetic material to allow for breed reconstruction.

Semen and embryos have been both proposed as materials for cryopreservation of animal genetic resources $[3,4]$. The amount of genetic material to be stored is a function of (among other factors) the intended future use of the material, which may include creation of new lines and breeds, supporting populations conserved in vivo, and breed reconstruction, with the latter generally being considered as the maximum guarantee against risk of breed loss. The literature on the type and amount of material to be stored as a function of the species and the conservation objectives is scant. Estimates for the number of semen doses or, alternatively, for embryos are given for some species by FAO [4], Ollivier and Renard [13], and Lömker and Simon [11], who also suggested parallel storage of embryos and semen. Results of an analysis for the creation of a pig semen bank were presented by Labroue et al. [9]. An overview of practical rules for collecting semen and embryos and of the organisational aspects of cryobanking setting were presented in a Workshop organised by the European Regional Focal Point (ERFP) for Animal Genetic Resources of the FAO [14].

Semen can be collected and frozen easily for most species. Breed reconstruction with semen-only can be accomplished through a series of back-cross generations, but this approach has some limitations, including the fact that one can never recover $100 \%$ of the genetics of the original breed. Gene percentage can be increased marginally by increasing the number of back-cross generations, but the number of doses of semen needed also increases, in some cases exponentially [13]. In addition, when the female reproductive potential is low (e.g. cattle, horse), the number of semen doses required can be very high. The number of semen doses (D) for breed reconstruction can be computed as

$$
D=d_{p} \times F \times n_{p},
$$

where $d_{p}$ is the number of doses needed per parturition, $F$ is the total number of females to be inseminated during the reconstruction process and $n_{p}$ is the expected number of parturitions per female [13]. For example, to reconstruct a horse population of 25 females, with $97 \%$ of the original genome, the number of semen doses required could exceed 20000.

The techniques for embryo collection and freezing are continually improving, but cryopreservation with embryos is still generally more complex, difficult, and costly than with semen. In addition, the collection of a sufficient quantity of embryos for complete reconstruction may require a number of donor females that is unlikely to be available for some endangered breeds. 
For example, Ollivier and Renard [13] suggested the storage of 300 unsexed cattle embryos from up to 90 cows. For many rare breeds, finding 90 donor females would be a difficult, if not impossible task.

The combined use of semen and embryos could allow one to overcome the restrictions associated with the cryopreservation of semen or embryos only. In particular, this strategy would allow the retention of $100 \%$ of the original genome of the breed to be reconstructed and, depending on the species considered, may reduce both the numbers of doses and donors when compared to the semen-only and embryo-only strategies. Except for the general suggestion from Lömker and Simon [11] to conserve both semen and embryos, no reports are available in the literature on the combined use of semen and embryos.

The objective of this study was to use a simulation to evaluate the use of a combination of semen and embryos in the creation of gene banks for reconstruction of an extinct breed, on the basis of the amount of material to be stored, time for reconstruction, maintenance of genetic variability and extinction probability during reconstruction.

\section{MATERIALS AND METHODS}

\subsection{Simulation}

The simulation was designed to model the amount of genetic material, semen and embryos, to be cryopreserved for reconstructing a population of 25 males and 25 females of reproductive age. A population of this size can guarantee an effective population size of 50 or higher, which is above the size considered as critical for the maintenance of genetic variation $[4,12]$. The material stored in the gene bank was assumed to be derived from at least 25 unrelated founders consisting of the donor sires (semen) plus the sires and dams of the embryos. The use of at least 25 founders guarantees retention (in the stored material) of $98 \%$ of the heterozygosity of the donor population, a value considered as an acceptable compromise between costs and conservation objectives $[4,15]$.

The basic scheme of the simulation is shown in Figure 1. The starting point of each simulation was assumed to be an existing gene bank consisting of a certain number of frozen embryos. Then, for each embryo, the probability of pregnancy, survival of the embryo and recipient to parturition, sex of the embryo, and survival to breeding age were simulated. The probabilities of each of these events are presented in Table I. These values were taken from the analyses of survival in commercial populations (our unpublished data) and consultation 


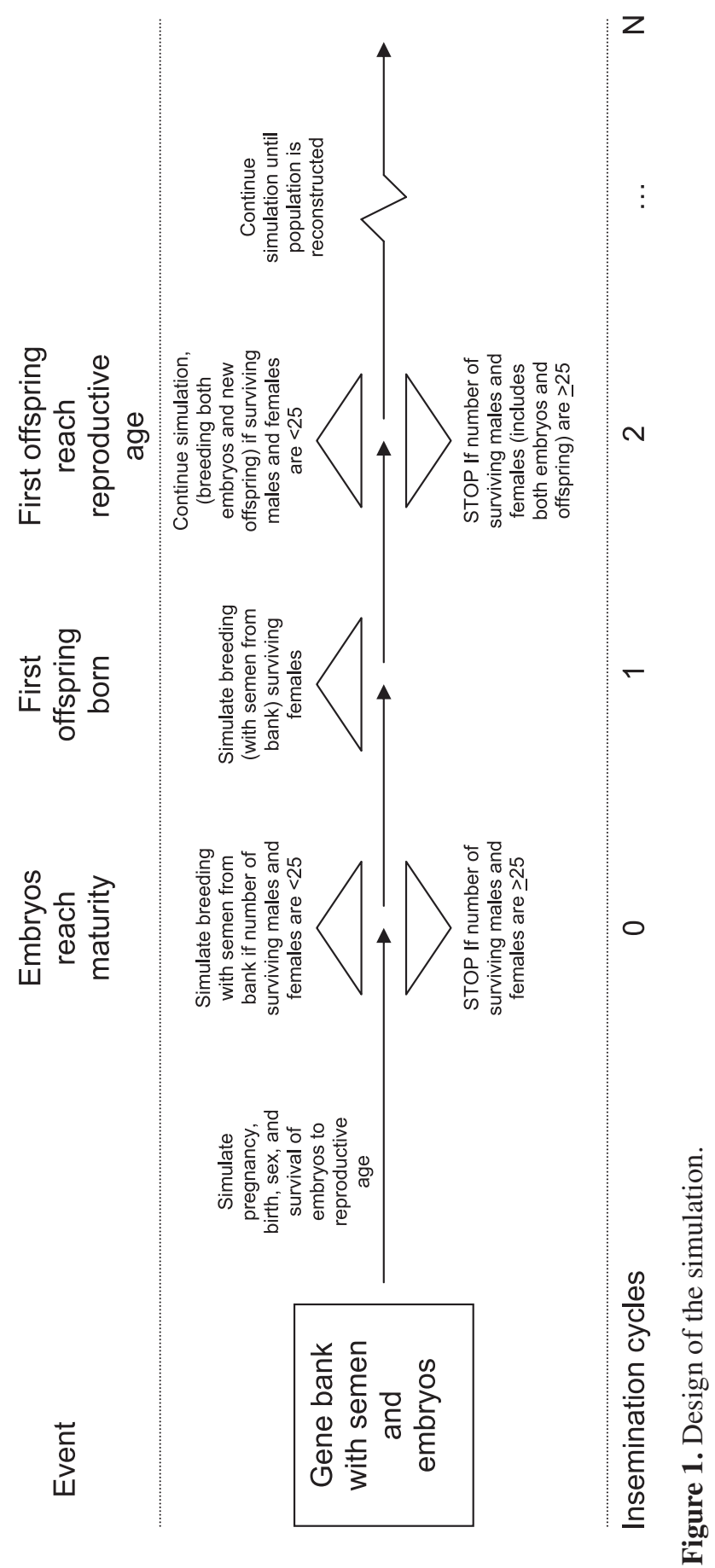


Table I. Reproductive and survival parameters common to all simulated species ${ }^{1}$.

\begin{tabular}{ll}
\hline Parameter & Value \\
\hline Proportion of females & 0.50 \\
Conception rate (semen) & 0.50 \\
Pregnancy rate (embryos) & 0.40 \\
Survival from birth to breeding age / conception & 0.80 \\
Survival from conception to parturition & 0.90 \\
Survival from first to second parturition & 0.86 \\
From 2nd to 3rd & 0.80 \\
3rd to 4th & 0.75 \\
4th to 5th & 0.75 \\
5th to 6th & 0.70 \\
6th to 7th & 0.70 \\
7th to 8th & 0.65 \\
8th to 9th & 0.50 \\
9th to 10th & 0.00 \\
\hline
\end{tabular}

${ }^{1}$ Estimated from different sources, technical reports and field experts.

with experts in embryo transfer (J. Metzger, personal communication, 2004). If the simulated population of embryos surviving to breeding age consisted of at least 25 females and 25 males, the breed was assumed to be "reconstructed" and a new replicate was initiated, starting from the gene bank stage. If the number of surviving embryos was less than 25 for either sex, the subsequent insemination of the female survivors with semen stored in the bank was simulated. In a reconstruction plan, semen usage must remain relatively balanced across sires because high variability in the size of half-sib families will contribute to a decreased effective population size in the reconstructed population [7, 8]. In order to help distribute sires evenly across animals, bulls were used sequentially. Conception was simulated with a probability of success of 50\% (Tab. I). If conception was not initially successful, up to five attempts at rebreeding were allowed. The animals that did not conceive after five inseminations were culled. The insemination of animals surviving from the original embryos was considered to be the start of the first "insemination cycle", a term that was used in this study to measure time for reconstruction and make comparisons across gene bank designs. An insemination cycle started at the time of insemination for the birth of one set of offspring, and ended at the insemination to produce the subsequent set. Once conception was obtained, the subsequent survival of the pregnant animals to parturition was then simulated.

Four types of species were considered, which varied according to mean and variability of family size: (i) a lowly prolific species producing a single 
Table II. Parameters describing the reproductive efficiencies of the four simulated species.

\begin{tabular}{lccc}
\hline \multirow{2}{*}{ Species type } & Examples & \multicolumn{2}{c}{ Number of offspring } \\
\cline { 3 - 4 } & & Mean & $\begin{array}{c}\text { Standard } \\
\text { deviation }\end{array}$ \\
\hline Single offspring & Cattle and horses & 1.0 & 0.0 \\
Twins & Small ruminants & 1.4 & 0.5 \\
Enhanced reproduction & Superovulated cattle & 2.6 & 2.0 \\
Litter bearing & Swine and rabbits & 7.5 & 2.5 \\
\hline
\end{tabular}

offspring per mating (SNG), (ii) a species for which a certain proportion of twinning (TWN) is expected, (iii) a category designed to simulate the use of a reproductive technology such as multiple ovulation and embryo transfer to increase the fecundity of a lowly prolific species (MET), and (iv) a highly prolific, litter-bearing (LIT) species. Table II lists examples of species for each situation and the means and standard deviations of number of offspring per female.

The animals that survived to produce offspring were then rebred, starting another insemination cycle to produce a second group of offspring. At the same time, the survival of the first group of offspring to reproductive age was simulated. At the time that the second group of offspring from the original embryos was born, the first group of offspring from these animals were assumed to have reached sexual maturity. The number of surviving females in these two groups ( 1 = original embryos and $2=$ their first offspring) was evaluated. If this total was at least 25 , the population was assumed to be "reconstructed" and a new replicate was started. If not, a new insemination cycle was generated and the mating of all these animals was simulated and the process was continued. At the end of each subsequent insemination cycle, the number of living animals of breeding age was counted to determine if the reconstruction goal of 25 animals from each sex had been reached. If not, additional insemination cycles were continually simulated. If the population could not be reconstructed in 50 cycles, it was assumed to have returned to a state of extinction. The simulation was replicated 500 times for each species type and each ratio of embryos to semen.

\subsection{Gene banks}

As already indicated, the simulated gene banks were assumed to be constructed of different combinations of semen and embryos. Each combination 
was based on having a given number of embryos, equal to a specific percentage of the embryos needed to reconstruct a breed using only embryos. For each combination, the number of embryos was fixed at the start of the simulation and the number of doses of semen was allowed to vary. The purpose of the simulation was thus to obtain the distribution of the number of doses of semen required to reconstruct the population for a particular number of embryos.

The first step in the preparation of the simulation was to determine the number of embryos needed to reconstruct a population using exclusively embryos. Because the primary goal of gene banks is to reduce the risk of extinction of a breed, the number of embryos used in this situation was not the expected number of embryos needed for construction (i.e. based on the average rates of conception and survival in Tab. I), but rather the number of embryos needed to reconstruct the population with a $90 \%$ (approximately) rate of certainty. Based on the parameters in Table I, the expected number of embryos needed to obtain 25 females of breeding age was 174. The approximate 90th percentile value for this variable was 215 embryos, which was constant for all species. (A detailed explanation of these calculations is in the Appendix).

For each combination of semen and embryos, the number of embryos in the bank was reduced by $10 \%$. Thus, the first situation simulated was for a bank with $90 \%$ of the embryos needed for exclusive-embryo reconstruction, or 194 embryos. The second combination simulated a bank with 172 embryos, and so forth, down to 22 embryos (10\%) in the final combination.

In addition to changing the numbers of embryos, the number of female donors of embryos was changed accordingly. The standard situation for reconstruction with $100 \%$ embryos called for 25 different female donors of embryos, and 0 male donors of semen [15]. Each time the number of embryos was decreased by $10 \%$, the number of female donors was decreased by this proportion as well, and replaced by male donors of semen. For the situation with $90 \%$ embryos, 22 donors of embryos and three donors of semen were simulated. With $80 \%$ embryos, the numbers of female and male donors were 20 and five, respectively, and so on.

\subsection{Comparison of gene banking strategies}

Five measures of efficiency of reconstruction were used to compare the banking strategies based on different proportions of embryos and semen. The first criterion was the risk of failure in the reconstruction. The risk was evaluated as the number of times that the new population failed to reach the size of 25 males and females of breeding age within 50 insemination cycles. 
The second criterion for evaluation was a measure of time required for reconstruction. For this study, time was measured in insemination cycles, to allow for application of the results across species.

The third measure of efficiency monitored was the number of doses of semen used in the reconstruction process, which are expected to increase for each incremental decrease in the number of embryos included in the bank. One criterion examined was the trend in the marginal increase in the number of doses of semen required for each $10 \%$ decrease in the number of embryos (from 90 to $10 \%$ ).

During the reconstruction process, a population is expected to lose some genetic variation, and to minimise this process should be an objective of any reconstruction strategy. Two criteria were used to monitor the genetic variability during the reconstruction process. The first was the additive genetic relationship among members of the reconstructed population. These values were averaged across the animals in the reconstructed population

$$
\mathrm{AA}=\sum_{i} \sum_{j \geq i}\left(a_{i j} / N\right),
$$

where AA is the average additive genetic relationship among animals, $a_{i j}$ is the additive genetic relationship between animals $i$ and $j$, and $N$ is the number of elements in the upper half of the additive genetic relationship matrix. It should be noted that for a given pair of animals $i$ and $j, a_{i j}$ is equal to 2 times the Malecot coefficient of kinship.

The second measure of the genetic variability maintained was the number of founder equivalents in the reconstructed population, $F_{e}[10]$. This statistic was calculated as

$$
\mathrm{F}_{\mathrm{e}}=\left[\sum_{i}\left(\sum_{j} a_{i j} / N_{r}\right)^{2}\right]^{-1},
$$

where $a_{i j}$ is the additive genetic relationship between founder $i$ and animal $j$ in the reconstructed population and founder $i$ and $N_{r}$ is the number of animals in the reconstructed population. The number of effective founders decreases as the level of disparity in the contributions of each founder to the final reconstructed population increases. It is known that $\mathrm{F}_{\mathrm{e}}$ has some limits, since it does not consider genetic drift and that after some generations, assuming random mating, becomes a constant value equal to half of the asymptotic effective population size [17]. Nevertheless, $\mathrm{F}_{\mathrm{e}}$ was considered appropriate for this study, given the very small number of generations of our populations and the absence of bottlenecks. Founders were defined as the sires and dams of 
embryos and the sires contributing semen to the gene bank; the actual number of founders ranged from 28 (22 sires with semen and six parents of embryos) with $10 \%$ embryos, to 47 (three sires with semen and 44 parents of embryos) with $90 \%$ embryos.

Minimising average relationships among reproductive individuals weighted by their progeny contribution is the strategy of choice [6] and approximate methods (e.g., $[2,16])$ and an exact method [5] have been proposed. Therefore, in addition to the sequential use of sires for homogenisation of genetic contributions (see Sect. 2.1), a strategy of sire distribution based on progeny contribution was also tested. For this approach, at the start of each insemination cycle, sires with semen in the gene bank were ranked for their average relationship with the reproductive population (lowest to highest). The first animal in the breeding population was then mated to the sire with the lowest level of relationship in the population. After that mating was made, a dummy animal with a relationship of 0.5 to the sire was added to the relationship matrix and the average relationship between the sires and the population was recalculated. The new sire with the lowest average relationship was then mated to the next animal to be bred. This process was continued until all animals were mated and repeated each insemination cycle until the population was reconstructed.

\section{RESULTS AND DISCUSSION}

\subsection{Risk of failure in the reconstruction}

Table III has the percentages of time that the reconstruction goal was not reached within 50 insemination cycles for each of the four species types. The risk of failure increased as the proportion of embryos decreased. For all species, the reconstruction goal was always reached when the embryo percentage was at least $40 \%$. In addition, the failure rate was higher at low reproductive prolificacy. Inasmuch as the goal of creating a genebank for breed reconstruction is to avoid extinction, construction of a bank with only $10 \%$ of the embryos required for an exclusive embryo bank seems to be an ill-advised and risky strategy. To be sure of reaching the reconstruction goal, embryo percentages should be higher than $30 \%$ for SNG and higher than $20 \%$ for more prolific species.

\subsection{Time for reconstruction}

Figure 2 shows the median (2A) and upper 90th percentile (2B) for the number of insemination cycles required to reconstruct each of the four species types 
Table III. Risks (\%) of failure during the breed reconstruction process for each type of species and for different proportions of embryos in the bank. Successful reconstruction was always observed for embryo percentages $\geq 40$.

\begin{tabular}{lccc}
\hline & \multicolumn{3}{c}{ Embryo percentage } \\
\cline { 2 - 4 } Species type $^{1}$ & 10 & 20 & 30 \\
\hline SNG & 12.0 & 1.0 & 0.4 \\
TWN & 6.6 & 0.4 & 0.0 \\
MET & 4.8 & 0.2 & 0.0 \\
LIT & 3.6 & 0.2 & 0.0 \\
\hline
\end{tabular}

${ }^{1} \mathrm{SNG}=$ single offspring per pregnancy, $\mathrm{TWN}=$ twinning possible, $\mathrm{MET}=$ use of multiple ovulation and embryo transfer, LIT $=$ litter bearing species.

$2 \mathrm{~A}$

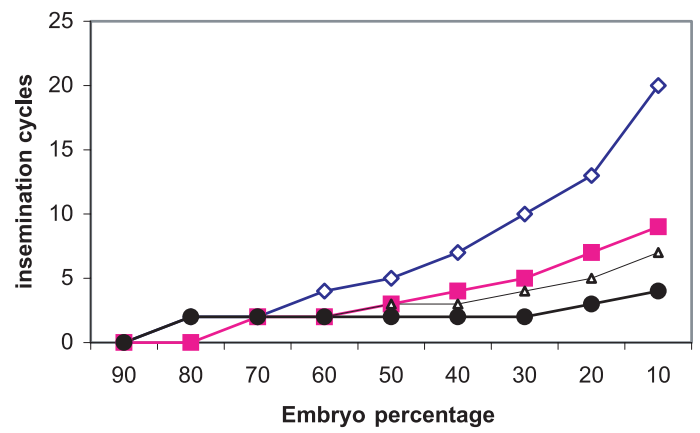

$\diamond$ SNG - TWN $₫$ MET $\multimap$ LIT

$2 \mathrm{~B}$

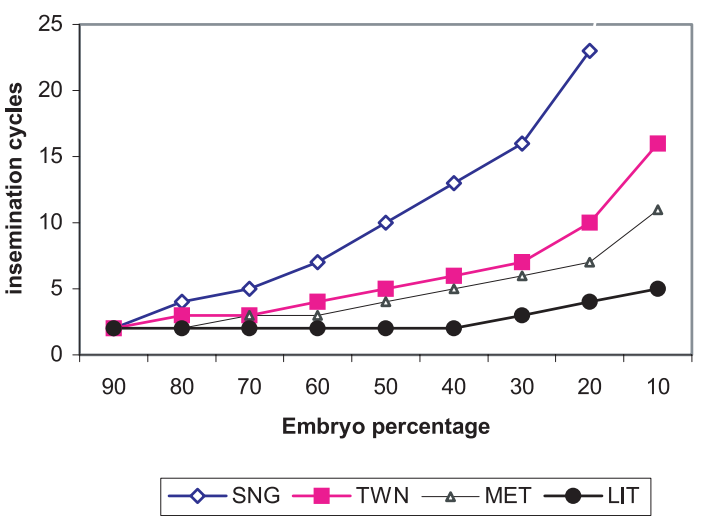

Figure 2. Median (A) and 90th percentile (B) for the numbers of insemination cycles required to reconstruct a breed when the number of embryos stored in the gene bank ranged from 90 to $10 \%$ of that required to reconstruct the population with only embryos, for four types of livestock species. 
for the different combinations of embryos and semen ranging from 90 to $10 \%$ embryos. The time for reconstruction increased with decreasing proportions of embryos. As explained previously, the benchmark value for a bank of $100 \%$ embryos was calculated on the objective to obtain reconstruction $90 \%$ of the time, and was equal to 215 embryos. For that reason, when only $90 \%$ of the benchmark value of embryos was used, reconstruction was still reached with the embryos only in most of the simulations, and thus required zero insemination cycles. As the proportion of embryos decreased, so did the proportion of times that the population was reconstructed from only embryos. With $80 \%$ of the benchmark, reconstruction was still achieved about $50 \%$ of the time. In some rare cases $(<1 \%$ of the time), the reconstruction goal was reached with only the offspring from the embryos even when the bank included only $50 \%$ of the benchmark.

A strong relationship was observed between fecundity and number of cycles required for reconstruction. For LIT species, the median time for reconstruction was always less than four cycles, even when only $10 \%$ of the benchmark embryos were used. In contrast, $>20$ cycles were required for reconstruction of SNG species when only $10 \%$ of the benchmark was used. With low prolificacy, the number of cycles increased exponentially as the percentage of embryos decreased. The results for the TWN and MET species fell between these two extremes. In general, fewer than ten cycles were required at these levels of prolificacy, even for the lowest proportions of embryos. Decreased fecundity also increased the variability of reconstruction times. For LIT species, the result for the 90th percentile was always either the same as the median or greater by only a single cycle. For TWN and MET, the 90th percentile was generally 130 to $150 \%$ of the median. For SNG, the 90th percentile was usually about two times the median. For an embryo percentage of $10 \%$, failure to reconstruct the SNG population occurred more than $10 \%$ of the time (Tab. III) and thus the $90 \%$ percentile was not calculable.

\subsection{Doses of semen required}

The number of doses of semen required followed the same general trends observed for a number of insemination cycles (Fig. 3). Specifically, as prolificacy increased, the number of doses of semen required decreased. The trend in the number of semen doses required when decreasing the number of embryos in the bank by $10 \%$ was basically linear for LIT, MET and TWIN, with marginal increases of 1.2, 5.0, and 10.2 doses per 10\% decrease in embryos, respectively. For SNG, the trend was not linear throughout its range, seemingly 


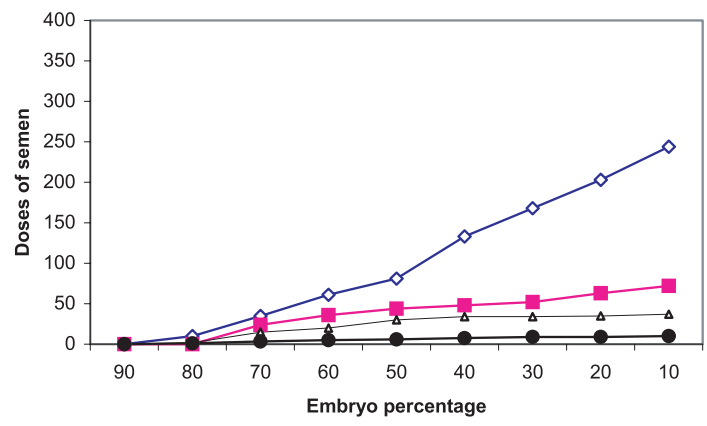

$\leadsto$ SNG $\rightarrow-$ TWN $\neg-$ MET $\multimap$ LIT

3B

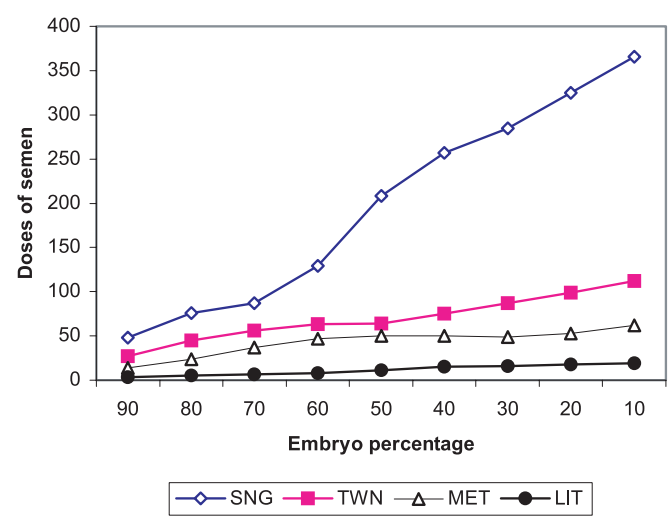

Figure 3. Median (A) and 90th percentile (B) for the numbers of doses of semen required to reconstruct a breed when the number of embryos stored in the gene bank ranged from 90 to $10 \%$ of that required to reconstruct the population with only embryos, for four types of livestock species.

having one marginal rate of about 24 doses per $10 \%$ reduction from 80 to $50 \%$ and about 41 doses per $10 \%$ below $50 \%$.

In practice, artificial insemination for LIT and MET often involves the administration of two doses of semen, separated by an interval of several hours. This approach of insemination with two doses of semen was not accounted for in Figure 3, but semen usage would have still been much lower than for SNG and TWN. No other estimates of combinations of embryos and semen for breed reconstruction are available in the literature, other than the broad guidelines discussed by Brem et al. [1] and Lömker and Simon [11]. The latter 
authors suggested the storage of 300 embryos and 2500 doses of semen to reconstruct a population of 25 cows, which are quantities much higher than our findings. However it should be noted that these authors suggested breed reconstruction by embryos only and storage of semen only for its subsequent reproduction.

\subsection{Maintenance of genetic variability}

Trends in the level of genetic relationships among members of the reconstructed population are shown in Figure 4. In general, with decreased embryo percentage and increased numbers of insemination cycles, relationships in the reconstructed population increased. In contrast to the trends observed for numbers of insemination cycles and doses of semen, less difference was observed among the four species types. In particular, the levels of relationships in the final population were very similar for SNG, TWN, and MET, at least for embryo percentages higher than $10 \%$. With increased fecundity, the number of full-sib groups is increased, while the number of half-sib relationships is greater for species with low fecundity. These two factors seemed to be in equilibrium for SNG, TWN, and MET. In contrast, the level of relationships with LIT tended to be lower than for the other three species types with moderate embryo percentages (40 to $70 \%$ ), but higher when embryo percentages were low (10 and $20 \%$ ). With low embryo percentages, the reconstructed LIT populations were made up of multiple full- and half-sib families from a few dams, plus some of the dams of these families. As a result, median levels of relationships were very high, up to 0.17 with $10 \%$ embryos in the bank.

The numbers of effective founders in the final population decreased by decreasing embryo percentage and, as a result, by increasing the number of cycles (Fig. 5). This result was as expected, inasmuch as the effective number of founders is conceptually related to kinship. As was observed for the level of relationships, trends were similar for SNG, TWN, and MET, and for LIT for embryo percentages greater than $40 \%$. As mentioned previously, the actual number of founders was greater for the situations with higher embryo percentages, because each embryo had two parents as founders, while each donor of semen was counted as a single founder. With an embryo percentage of $10 \%$, the effective number of founders was near 10 for LIT, but remained higher than 15 for SNG, TWN and MET. As observed in Figures 4 and 5, increased levels of relationships among members of the reconstructed population are a factor that could potentially limit the applicability of gene banking strategies that rely on using few embryos and large proportions of semen. As demonstrated, such 


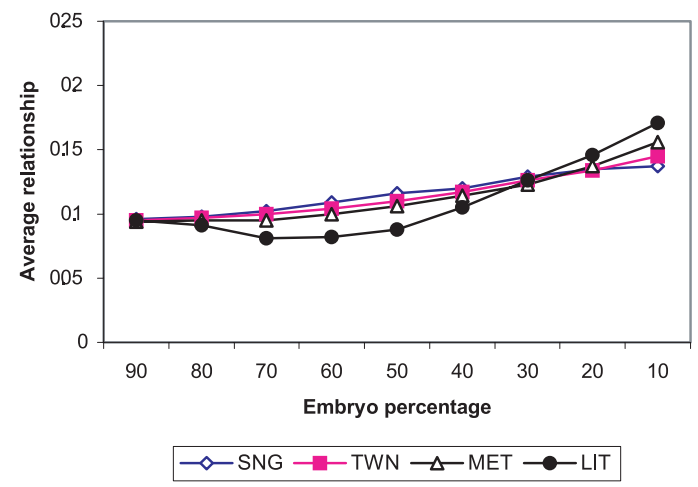

4B

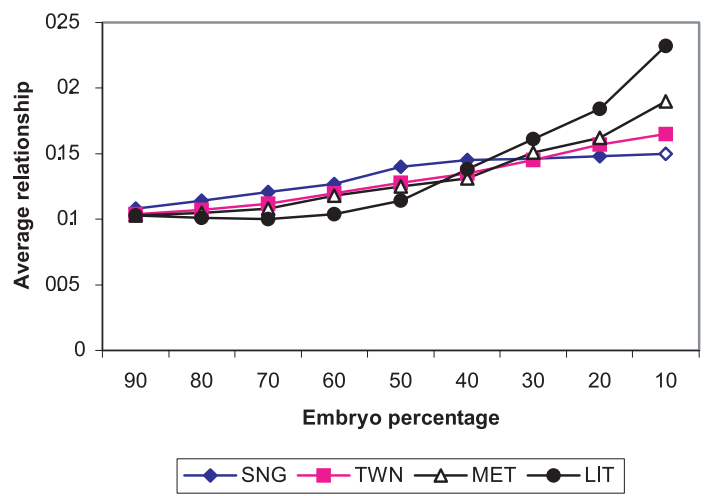

Figure 4. Median (A) and 90th percentile (B) for the average genetic relationship among members of a reconstructed breed when the number of embryos stored in the gene bank ranged from 90 to $10 \%$ of that required to reconstruct the population with only embryos, for four types of livestock species.

strategies require numerous insemination cycles and create multiple half- and full-sib families.

Figure 6 shows the effect of applying a strategy to minimize genetic ties on the mean level of relationships in reconstructed SNG populations, as well as the mean level of relationships obtained using the passive, sequential method of sire usage. The use of the strategic mating approach resulted in lower levels of relationships for all embryo percentages, and the advantage of the method increased as embryo percentage decreased. Using this method, the average relationship with $10 \%$ embryos was about the same as was observed with $40 \%$ 
$5 \mathrm{~A}$

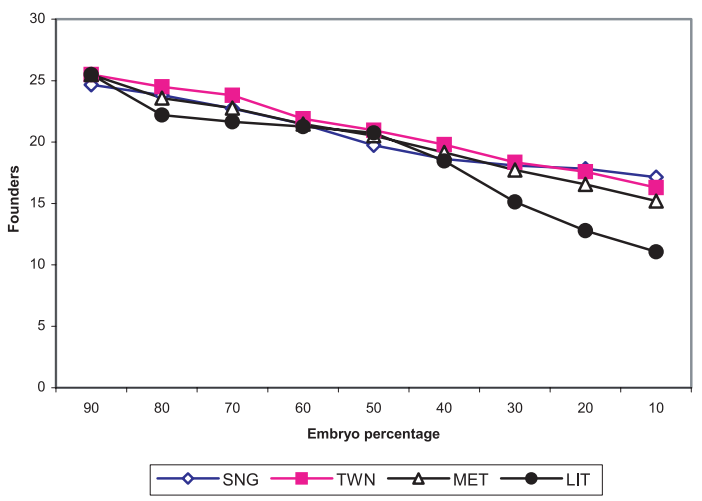

$5 \mathrm{~B}$

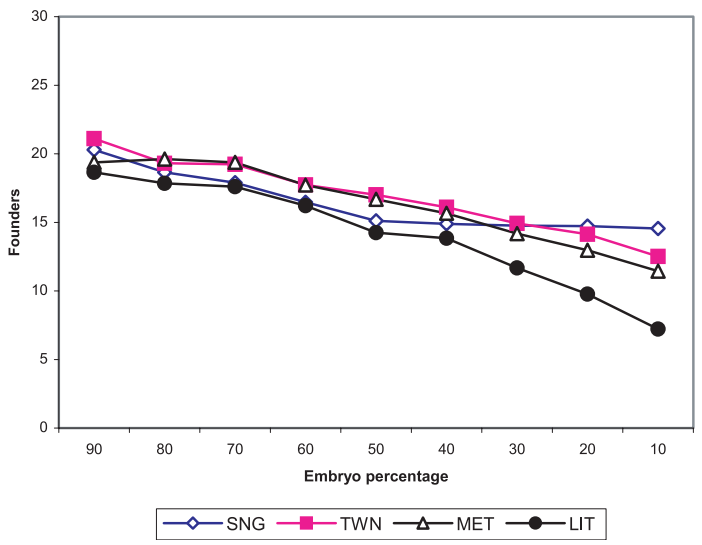

Figure 5. Median (A) and 90th percentile (B) for the average number of founders among members of a reconstructed breed when the number of embryos stored in the gene bank ranged from 90 to $10 \%$ of that required to reconstruct the population with only embryos, for four types of livestock species.

embryos and the sequential method of sire distribution. The number of effective founders was greater than that obtained when applying the passive method to an embryo percentage of $60 \%$ (not shown). Thus, the use of strategies that optimise genetic contributions or minimise genetic relationship can improve the feasibility of applying strategies using relatively low proportions of embryos. 


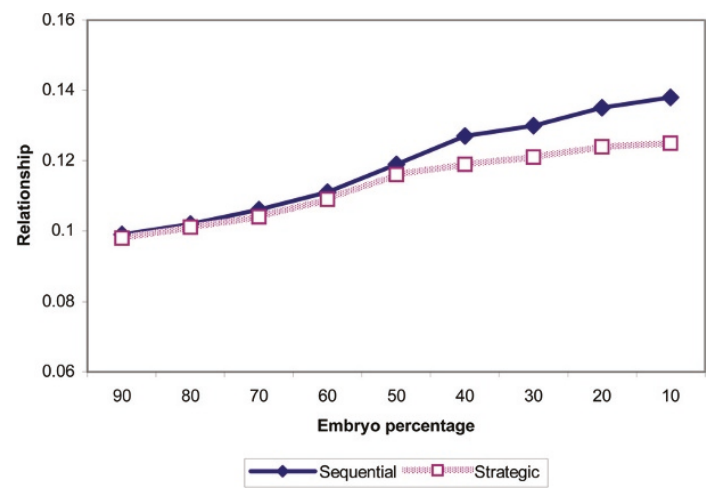

Figure 6. Means (for proportions of embryos ranging from 90 to 10\%) of additive genetic relationships among members of the reconstructed population of a species with single offspring when the sires are used sequentially or strategically to equalise their contribution to the final population.

\subsection{Final considerations}

The objective of this study was to investigate a new approach for the creation of gene banks for breed reconstruction, by using embryos and semen in combination. Further investigation is in progress to extend the comparison of this approach with the semen-only and embryo-only strategies generally advocated to consider the costs associated with each strategy. Such a comparison requires the analysis of several aspects not considered in the current work. However, some preliminary and general indications on costs can be derived from the results of this study. For example, the SNG situation was that for which the ratio of substitution of semen for embryos was the highest. Decreasing the percentage of embryos from $40 \%$ to $30 \%$ reduced the number of embryos needed by 22 , from 86 to 65 . In turn, the number of doses of semen needed increased by about 40 (Fig. 3A). Therefore, each embryo withheld from the bank can be replaced by two doses of semen. Using a very simplistic logic, one can conclude that substituting embryos with semen will be economically favourable as long as each embryo costs more than twice the price of a dose of semen. Currently reasonable estimates for the costs to obtain a freezable embryo and a unit of semen are $100 €$ and $1.5 €$, respectively. This ratio is approximately 67 , which is far superior to 2 , thus favouring strategies with a higher proportion of semen. Of course, this was a simple example and other factors must be considered when constructing the genebank. Using the same example, the median number of insemination cycles was seven with $40 \%$ embryos and 10 with 30\% embryos (Fig. 2A). For cattle, each reproductive cycle 
would last about one year, so decreasing the number of embryos also increases the time for reconstruction. Creating a gene bank would have to balance initial monetary costs with the desire to reach the reconstruction goal in the most timely manner.

\section{CONCLUSIONS}

Erosion of genetic resources in many parts of the world is advancing at high speed and in many cases in situ conservation strategies are not sufficient because they often require years to be put into effective operation. The results from the simulations in this study provide general guidelines for the creation of cryogenic gene banks. Numerous efforts for the banking of semen and embryos have been recently started in many countries, but there remains a need to complete them. The results of this study also provide information that can be used to optimise the completion of partially existing banks.

The design of the most efficient cryo-banking system will require consideration of costs, and that research is on going. However, some specific recommendations on other aspects of design can be made based on the results of this study. First, to ensure that the reconstruction goal will be reached, embryo percentages should be $>30 \%$ for SNG and $>20 \%$ for other species. In addition to increasing the risk of failure in the reconstruction, decreasing the embryo percentage also tends to increase the level of relationships among members of the reconstructed population. However, strategic mating plans can help alleviate this problem and should, therefore, be applied when the embryo percentage is less than $50 \%$.

\section{ACKNOWLEDGEMENTS}

This research work was funded in part by a grant from the Region of Lombardy, Italy. The authors thank George Wiggans, Ken Stalder, and John Metzger for data used to obtain parameters underlying the simulation (Tab. I).

\section{REFERENCES}

[1] Brem G., Graf F., Kräusslich H., Genetic and economic differences among methods of gene conservation in farm animals, Livest. Prod. Sci. 11 (1984) 65-68.

[2] Brisbane J.R., Gibson J.P., Balancing selection response and rate of inbreeding by including genetic relationship in selection decisions, Theor. Appl. Genet. 91 (1995) 421-431. 
[3] ERFP, Guidelines for the constitution of national cryopreservation programmes for farm animals, Hiemstra S.J. (Ed.), Publication No 1 of the European Regional Focal Point on Animal Genetic Resources, 2003.

[4] FAO, Secondary Guidelines for Development of National Farm Animal Genetic Resources Management Plans, FAO, Rome, Italy, 1988.

[5] Fernandez J., Toro M.A., The use of mathematical programming to control inbreeding in selection schemes, J. Anim. Breeding Genet. 116 (1999) 447-466.

[6] Fernandez J., Toro M.A., Caballero A., Managing individuals' contributions to maximize the allelic diversity maintained in small, conserved populations, Cons. Biol. 18 (2004) 1358-1367.

[7] Hill W.G., A note on effective population size with overlapping generations, Genetics 92 (1979) 317-322.

[8] Kimura M., Crow J.F., The measurement of effective population number, Evolution 17 (1963) 279-288.

[9] Labroue F., Loquet M., Guillouet P., Bussière J.J., Glodek P., Wemheuer W., Gandini G., Pizzi F., Delgado J.V., Poto A., Peinado B., Sereno J.R.B., Ollivier L., Pig Semen banks in Europe, in: Characterisation and conservation of pig genetic resources, in: Ollivier, Labroue, Glodek, Gandini and Delgado (Eds.), EAAP Publication No. 104, Wageningen Pers, Wageningen, 2001.

[10] Lacy R.C., Analysis of founder representation in pedigrees: founder equivalents and founder genome equivalents, Zoo. Biol. 8 (1989) 111-123.

[11] Lömker R., Simon D.L., Costs of and inbreeding in conservation of endangered breeds of cattle, 5th World Congr. Genet. Appl. Livest. Prod. 21 (1994) 393-396.

[12] Meuwissen T.H.E., Woolliams J.A., Effective sizes of livestock populations to prevent a decline in fitness, Theor. Appl. Genet. 89 (1994) 1019-1026.

[13] Ollivier L., Renard J.P., The costs of cryopreservation of animal genetic resources, Proc. 53rd Ann. Meet. Eur. Assoc. Anim. Prod., 1995, Wageningen Academic Publishers, Wageningen, The Netherlands.

[14] Planchenault D., Workshop on cryopreservation of animal genetic resources in Europe, February 23, 2003, Paris, France.

[15] Smith C., Estimated costs of genetic conservation of farm animals, in: FAO Animal Production and Health Paper 44/1, 1984, FAO, Rome, pp. 21-30.

[16] Wray N.R., Goddard M.E., Increasing long-term response to selection, Genet. Sel. Evol. 26 (1994) 431-451.

[17] Wray N.R., Thompson R., Prediction of rates of inbreeding in selected populations, Genet. Res. 55 (1990) 41-54.

\section{APPENDIX}

The expected number of embryos needed to obtain 25 females of breeding age, $E\left(n_{25}\right)$, was calculated according to the following formula:

$$
E\left(n_{25}\right)=25 /(c \times s r \times f \times s b),
$$


where, $c$ is the probability of implantation of the embryo $(c=0.4), s r$ is the probability of survival of the recipient until parturition $(s r=0.9), f$ is the probability that the embryo was female $(f=0.5)$, and $s b$ is the probability of survival of the embryo from birth to breeding age $(s b=0.8)$. Based on these proportions, $E\left(n_{25}\right)=174$. The number of females $\left(n_{f}\right)$ obtained was assumed to be distributed binomially with mean equal to 25 and variance equal to

$$
\sigma^{2}\left(n_{f}\right)=E\left(n_{25}\right) \times D \times(1-D),
$$

where $D$ is equal to the denominator of equation (1). A normal approximation to the binomial distribution was applied to obtain the 90th percentile of the distribution of $n_{f}, E 90\left(n_{25}\right)$ :

$$
E 90\left(n_{25}\right)=25+1.28 \times \sigma\left(n_{f}\right),
$$

where 1.28 is the $Z$ value corresponding to the 90th percentile of the normal distribution. This value $\left[E 90\left(n_{25}\right)=31\right]$ was then divided by $D$ from equation (2) to obtain the number of frozen embryos that, when thawed and implanted in recipients, would result in $\geq 25$ females of breeding age with a $90 \%$ probability. The result of these calculations was 215 embryos.

To access this journal online: www.edpsciences.org 